\title{
Virtopsy-The Scalpel Free Autopsy: A Review of Literature
}

\author{
Amita Rahul Navalkar
}

\section{ABSTRACT}

Forensic medicine aims for the documentation of medical and other forensic findings in living and deceased persons for the police and the judiciary system. Autopsy is the scientific examination of bodies after death, where whole surface of the body as well as all the body cavities are explored to record the findings. At the same time it is also equally important to consider the sentiments of the relatives of the deceased who may get upset at conventional autopsies. So, if there exists a means by which all the findings in the body can be established without hurting anyone's sentiments should be accepted. Virtopsy or virtual autopsy is one step ahead in this field where three-dimensional (3D) scans using computed tomography (CT) scans and magnetic resonance imaging (MRI) pictures are employed sans using the scalpel for performing autopsies on victims to receive more information aiding in diagnosis and contributing significantly toward forensic science. This review wishes to highlight virtopsy as an important tool in forensic imaging and identification.

Keywords: Forensic imaging, Photogrammetry, Scalpel free autopsy, Virtopsy, Vitrobot.

How to cite this article: Navalkar AR. Virtopsy-The Scalpel Free Autopsy: A Review of Literature. J Contemp Dent 2015;5(3):168-172.

Source of support: Nil

Conflict of interest: None

\section{INTRODUCTION}

'There is nothing like an Autopsy for Prognosis.'

Autopsy the scientific examination of bodies after death, where whole surface of the body as well as the body cavities are explored to record findings, has been the mainstay in all forensic investigations.

The main objective of forensic medicine is to document, analyze and elucidate scientific medical findings in a comprehensive way in both living as well as deceased individuals for courtroom presentation. In a deceased person, the main goals are to determine the cause of death and the manner of death to evaluate the vitality of the sustained injuries and to develop a forensic reconstruction based on the findings.

\section{Professor}

Department of Oral Medicine, Diagnosis and Radiology, YMT Dental College and Hospital, Navi Mumbai, Maharashtra, India

Corresponding Author: Amita Rahul Navalkar, Professor Department of Oral Medicine Diagnosis and Radiology, YMT Dental College and Hospital, Kharghar, Navi Mumbai, Maharashtra India, Phone: 27564429, e-mail: amitanavalkar@gmail.com
Though highly unpleasant for the general public to think about, autopsies hold an important place in our society, providing an extremely crucial link between the deceased and that which killed them. As the knowledge and skills of scientists and doctors expands over the years, so does the techniques and armamentarium required increase in utility and sensitivity. ${ }^{1}$

Invasive body opening autopsy represents a traditional means of postmortem investigation. As worded by Brogdon. 'The sad truth is that more than a century after the first X-ray was introduced as evidence in a court of law, there is no general appreciation of the extent of the radiology potential in forensic science.

The concept of 'virtopsy' was thus established from the desire to implement new techniques in radiological imaging for the benefit of forensic science. It is meant to be an objective documentation and analysis process of physical features and evidence in a three-dimensional (3D) fashion. ${ }^{2}$

\section{HISTORY}

The first forensic application of computed tomography (CT) scan was a description of the pattern of a gunshot injury to the head by Wulleweber et al in 1977. Due to limited image quality and resolution and poor postprocessing results, only a few studies correlated pathologic findings at full body postmortem CT with forensic autopsy findings. ${ }^{3}$

In 1989, Kalender et al introduced spiral CT opening the door for 3D data acquisition and processing. However, this did not increase the interest of forensic scientists in this new modality.

The concept of objective, noninvasive documentation of the body surface for forensic purpose arose in the early 1990s with the development of photogrammetry.

In 2000, it was suggested that observer independent documentation of the body surface be combined with observer independent documentation of the interior of the body.

This has now been made possible by the virtopsy project of the Institute of Forensic Medicine, Diagnostic Radiology and Neuroradiology at the University of Bern, Switzerland by the team led by Richard Dirnhofer, Michael J Thali et al. Virtopsy is basically a scalpel-free autopsy carried out using modern medical imaging and measuring technology. Here, there is no need for 
physical dissection of the body. Different imaging techniques provide a complete $3 \mathrm{D}$ view of the inside as well as the outside of the body. According to Michael Thali, by performing a traditional autopsy the 3D geometry of the body gets destroyed. Using the cross-sectional imaging techniques, the same findings and much more can be demonstrated in a noninvasive way. ${ }^{4}$

\section{DEFINITION}

Whenever a photograph is taken, it gives a two dimensional display where only the length and breadth of the wound are displayed. However, the depth cannot be ascertained in this two-dimensional (2D) representation. Hence to understand this view, a 3D view is necessary.

What began as localizing the placement of the tip of a knife in a wound to the aorta using CT has become a near future tool for more effective and detailed forensic examination of corpses.

Virtopsy is a portmanteau of virtual and autopsy. The term virtopsy was created from the terms 'Virtual' derived from the Latin word 'virtus', which means 'useful', 'efficient and good'. The term autopsy is a combination of the classical Greek term, 'autos', meaning 'self' and 'Opsomei', meaning 'I see'. Thus autopsy means 'To see with one's own eyes'. This neologism created by Richard Dirnhofer, a Swedish Forensic Pathologist describes the innovative combining of power of the virtual world in the form of graphics and the usefulness of researchers and forensic science of the autopsy into a powerful technique to help investigators determine the circumstances surrounding an individual's death. ${ }^{5}$

It is a promising tool that complements the information and discoveries of investigators, doctors and forensic pathologist when used in combination with traditional autopsies.

\section{VIRTOPSY PROCESS}

The first step in performing a virtopsy is to prepare the body for imaging (Fig. 1). This was done previously by placing small disks along the surface of the body so that the surface scanner could align the surface scans with the interior scans. The disks created single points which were converted to a single cohesive image by the computer. ${ }^{6}$

Vitrobot, places the markers on the surface of the corpse. This makes the results of the Virtopsy more accurate and standardized. The markers are used by the computer processors to calibrate the surface scan of the corpse and match it to the later internal imaging process (Fig. 2).

After the markers are placed the vitrobot takes a $3 \mathrm{D}$ color model to the body. This scan uses stereoscopic cameras to capture the color image and a projector to cast a mesh pattern on the body. Once the image is created the picture can be manipulated on a computer screen so that the investigators can turn it and identify the tattoos. Vitrobot places tripods and cameras at various points around the body. The robot then glides over the body creating a 3D image. This process requires about 10 seconds. ${ }^{7}$

After the surface scan the body is brought to the CT and magnetic resonance imaging (MRI) labs double wrapped in blue bags through which $\mathrm{X}$-rays can pass and then laid on the CT, MRI table. The bag remains closed while the body is scanned to protect the privacy of the deceased, to maintain cleanliness in the room. A CT scan done produces 25000 images in 20 seconds, each one a slice through the body. Then an MRI is done.

Using coronary artery desease (CAD) style programs and ultra powerful graphic sensors the data is analyzed. Within 19 minutes data representing thin X-ray slices of the body are reconstructed by the computer into detailed images of bone and tissue. Different tissues, bodily substances and foreign objects absorb the X-ray scanners to varying amounts. These different absorption levels are rendered in a $3 \mathrm{D}$ visualization of different colors and opacities-air pockets are blue, soft tissues are beige, blood vessels are red and bone is white.

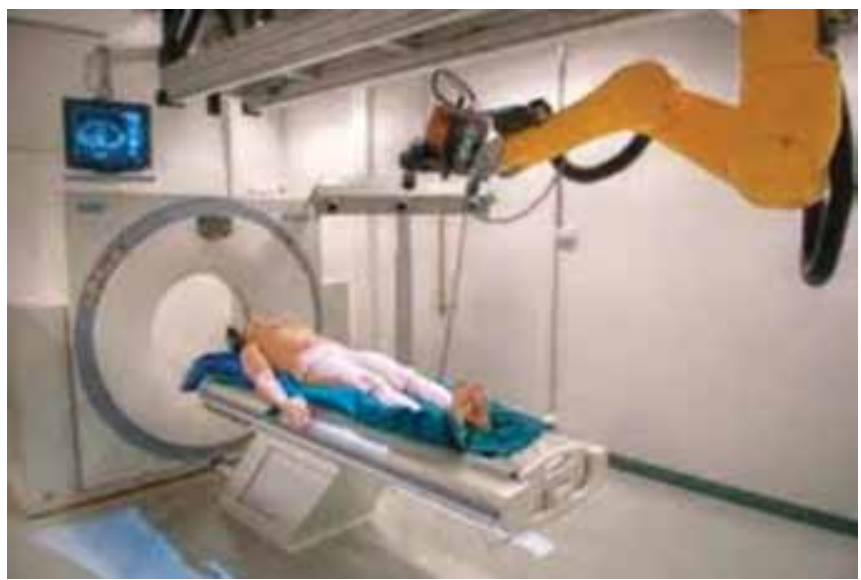

Fig. 1: Vitrobot

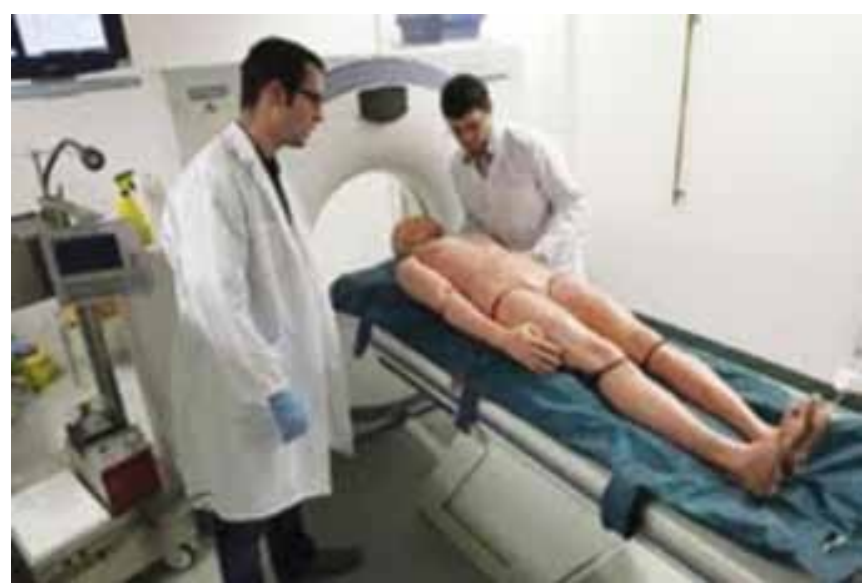

Fig. 2: Vitrobot scanning 
The pathologist can peel through layers of virtual skin using a computer mouse. Also the patterns and images can be manipulated up and down and turned to various angles. The vitrobot can also perform needle biopsies if body samples are required. All data scanners is then loaded and stored onto compact data disks.

Dr Anders Persson, Director of the Linköping University centre for medical imaging science and visualization, Sweden has created 'The Virtopsy Table'. This is a virtual autopsy table. A large touch sensitive liquid-crystaldisplay (LCD) screen represents the operating table displaying the image of the body. At the swipe of a finger, layers of skin and muscle are dissected. Also zooming in and out of the organs for their assessment and slicing through tissue using a virtual knife are possible (Figs 3 and 4).

\section{APPLICATIONS}

The role of forensic science is three fold, it assesses:

- Cause of death: The reasons or events that begins the process resulting in death (Fig. 8).

- Manner of death: Fashion or circumstance that results in death whether due to natural or unnatural causes, such as homicide, suicide or accident.

- Mechanism of death: Functional, physiologic or structural change that makes independent life no longer possible after a lethal event has occurred.

According to Thali, Dirnhofer, Vock (2009), virtopsy can determine manner of death whereas cause of death and mechanism of death are potential possibilities.

It thus can be used for,

- Vitality of sustained injuries: Vital reactions elucidate the sequence of injury and death in forensic pathologic evidence. The question of whether an injury was sustained before or after death is an important forensic matter, and the answer is provided for by forensic findings that occur with intact circulation, respiration, metabolism or consciousness. These are termed as vital reactions (Fig. 5).

- Forensic Reconstruction:

- Impact direction.

- Entrance and exit wounds (Fig. 6)

- Determination of automobile driver or airplane pilot.

- Medicolegal issues.

- Specific forensic findings, such as burnt corpses and putrified corpses.

- Bite mark registration and analysis (Fig. 7).

- Application of minimally invasive technique to enable collection of imaging guided biopsy technique for collection of tissue samples and also urine, bile, blood for toxicology or deoxyribonucleic acid (DNA) analysis.

- Application of microradiologic technique to analyze electrical injury patterns on human skin or to document specific ophthalmologic findings that might indicate 'shaken baby syndrome' when circumstances elude explanation.

- Morphologic fingerprints

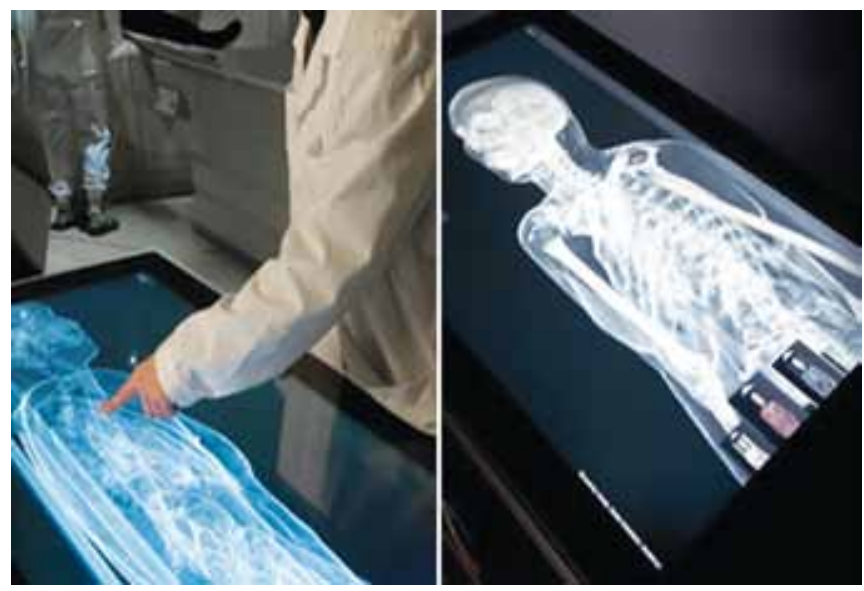

Fig. 3: Virtopsy table

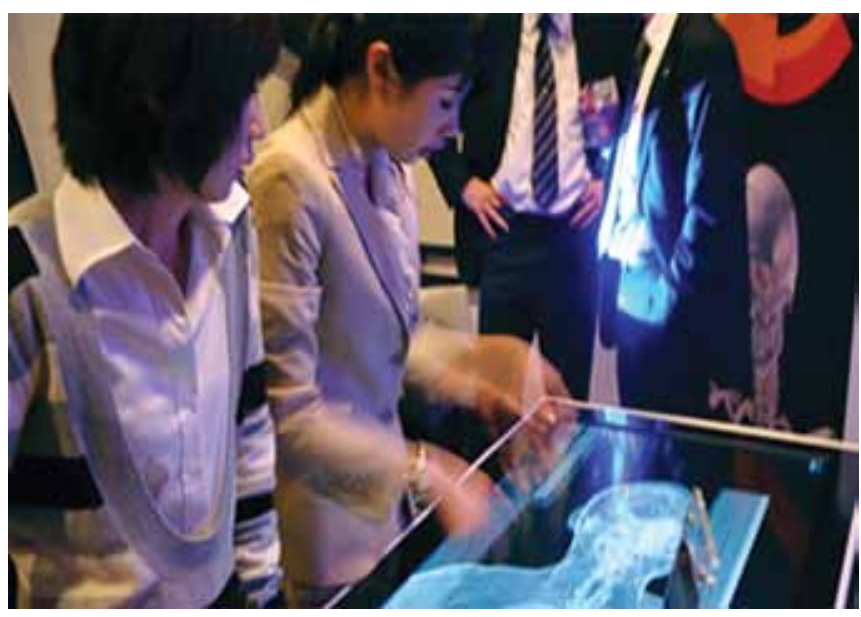

Fig. 4: Interactive virtopsy table

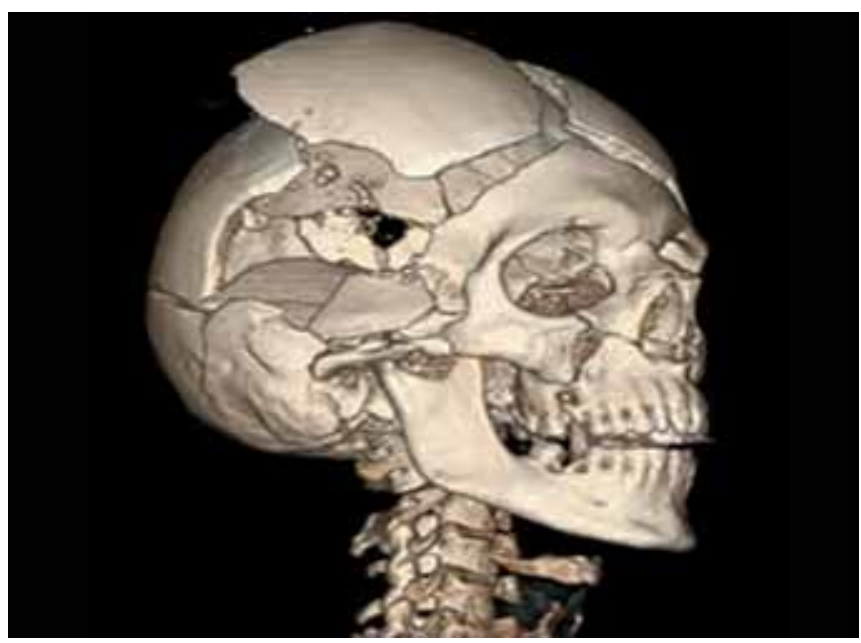

Fig. 5: Accident injury 


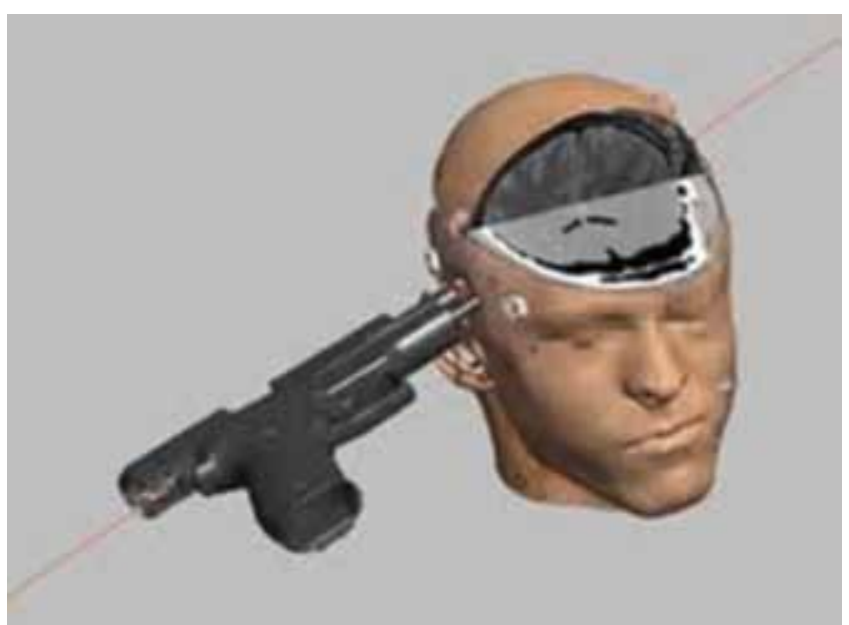

Fig. 6: Path of bullet entry and exit wound

- Real data based 3D forensic reconstruction of incidents.

- Forensic assessment of living persons.

- To assess the injury sustained to various organs resulting in the cause of death (Fig. 8).

\section{ADVANTAGES}

- It is most effective in study of wounds including matching of the probable weapon. In case of a Bullet injury, with a virtopsy the entry wound, tract of the bullet in the body as well as the exit wound can be assessed. Thus extensive dissection to trace the path of the bullet is avoided.

- No scalpel, hence no hazard of infection acquired by the mortuary staff as well as the concerned doctors as the procedures is bloodless.
- Body is not subjected to an incision. Hence for religious reasons the procedure is well accepted by the relatives of the deceased.

- The whole architecture of the body is preserved, so, if the body is subjected to a second autopsy, the mutilation of the body can be avoided

- The body can be viewed in layers from different angles for collection of evidence completely

- The $3 \mathrm{D}$, non-subjective information that is stored can be easily presented to the court. If necessary the data can be sent via CD or email to another forensic pathologist for a second opinion. It can be stored for future reference in the years to come if required. ${ }^{6}$

- It offers promise to many medical centers equipped with CT and MRI machines but lacking forensic pathologists.

- It promises to ease the burden of determining identity and cause of death of large number of victims following natural disasters, such as earthquakes and tsunamis.

- It helps to pinpoint the chain of events after a bomb blast or a terrorist attack.

\section{DISADVANTAGES}

- Small tissue injury may be missed.

- All pathological conditions may not be possible to diagnose.

- Cannot tell the color of organs especially when inflammation is suspected.
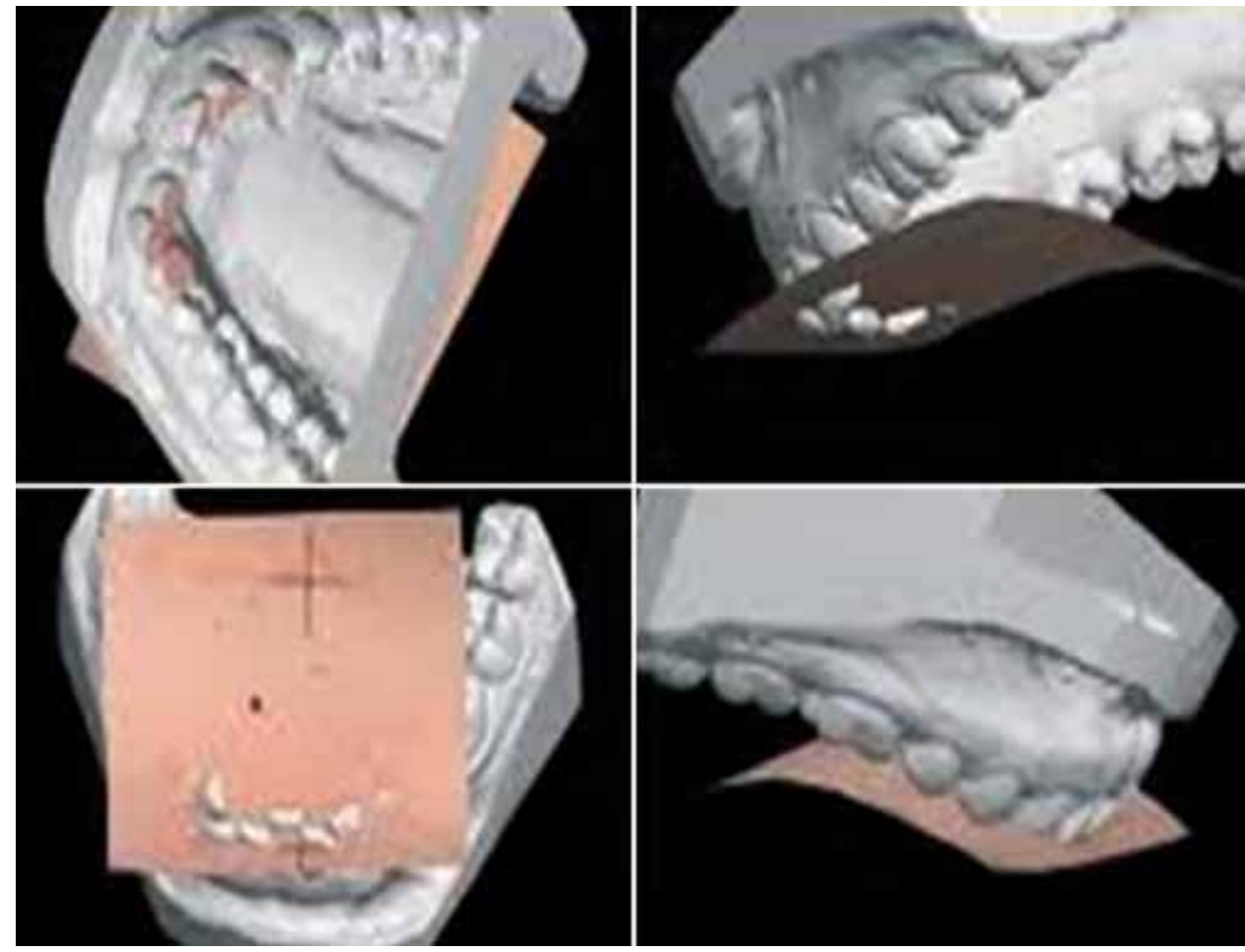

Fig. 7: Bite mark registration and analysis 


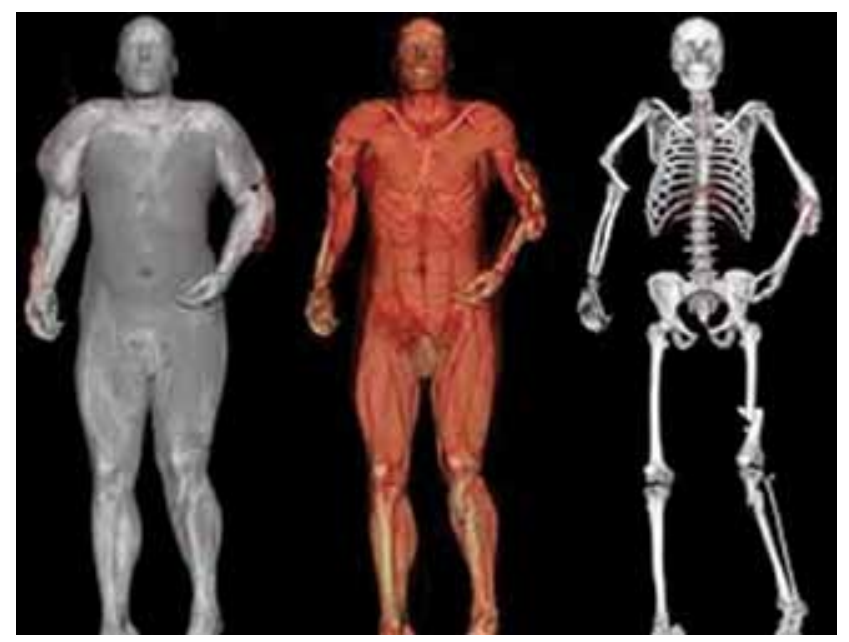

Fig. 8: Virtopsy of burn victims

- Cannot differentiate between pre death and post death injuries.

- Expensive.

\section{CONCLUSION}

As with any new technique there will be resistance or comments that question the validity and reasoning of the method. As the data concerning virtopsy increases in magnitude the benefits of the technique far outweigh the burdens of it.

It is important to note that the conventional autopsy has its own advantages which still enable it to be a modality of great effect in forensic sciences.

The Swiss pioneers who gave virtopsy to the world conclude that, with the use of imaging techniques, such as $\mathrm{CT}$ and MRI, photogrammetry and 3D optical measuring techniques a reliable, accurate geometric presentation of the forensic findings can be achieved.
Thus, in conclusion, the method of documenting forensic findings in a virtopsy are investigator independent, objective and noninvasive and will lead to a qualitative improvement in forensic pathologic investigation, since the digitally stored data can be recalled at any time to provide fresh, intact topographic and anatomic clinical information. Virtopsy combines very powerful scanning and radiographic technology with the power and resolution of modern computing. This makes it a tool which could tip the scales back to more regular use of the autopsy to help identify the manner and cause of death in individuals allowing researchers and investigators to discover important clues without the need to physically dissect the cadaver.

\section{REFERENCES}

1. Morgan Lynn. Virtopsy and the traditional autopsy. 9 th Nov 2010, www.wikinut.com

2. Thali MJ, et al. Virtopsy a new imaging horizon in forensic pathology: virtual autopsy by postmortem multislice CT (MSCT) and MRI: a feasibility study. J Forensic Sci 2003;48(2): 1-18.

3. Dirnhofer R, Jackowski C, Vock P, Potter K, Thali MJ. Virtopsy: minimally invasive, imaging guided virtual autopsy. RSNA 2006;26:1305-1333.

4. Patowary AJ. Virtopsy: One step forward in the field of forensic medicine: a review. J Indian Acad Forensic Med 30(1):32-36.

5. Thali M, Jackowski C, Oesterhelweg L, Ross SG, Dirnhofer R. Virtopsy-the swiss virtual autopsy approach. Leg Med (Tokyo) 2007 Mar;9(2):100-104.

6. Roychowdhury UB, Basak S, Gupta AK. Virtual autopsy: the future of forensic medicine www.indmedica.com;8(2):1-4.

7. Bixby J. Virtopsy: a new innovation for forensic science. International Association of Forensic Nurses: OTE16(3). Available at: www.iafn.org 